\title{
Rate of Convergence in Singular Perturbations of Hyperbolic Problems
}

\section{JOHN LAGNESE}

Communicated by the Editors

1. Introduction. Let $V_{\epsilon}, W_{\epsilon}, W$ and $X$ be Hilbert spaces ( $\epsilon$ being a real parameter, $\epsilon \boldsymbol{\varepsilon}(0,1]$ for example) with

$$
V_{\epsilon} \subset W_{\epsilon} \subset W \subset X .
$$

The inclusions are both algebraic and topological and each space is assumed dense in the one that follows it. The norm and scalar product in $X$ are denoted by $|\cdot|$ and $(\cdot, \cdot)$, respectively; norms in the other spaces are indicated with subscripts, for example $|\cdot|_{v_{e}}$.

We identify $X$ with its antidual. Then we have the usual inclusions

$$
X \subset W^{\prime} \subset W_{\epsilon}^{\prime} \subset V_{\epsilon}^{\prime}
$$

where the prime means antidual. If $x$ belongs to one of the spaces in (1.1) and $f$ to its antidual, we may, without abuse of notation, denote their scalar product in the antiduality by $(f, x)$; this coincides with their scalar product in $X$ when $f \varepsilon X$.

$t$ will denote a real variable taken from a finite interval $[0, T]$. For each $\epsilon$ and $t$ let $a_{\epsilon}(t ; u, v), b_{\epsilon}(t ; u, v)$ and $b(t ; u, v)$ be continuous sesquilinear forms on $V_{\epsilon}, W_{\epsilon}$ and $W$, respectively. There are bounded linear operators $a_{\epsilon}(t) \varepsilon$ $\mathfrak{L}\left(V_{\epsilon}, V_{\epsilon}^{\prime}\right), \bigotimes_{\epsilon}(t) \varepsilon \&\left(W_{\epsilon}, W_{\epsilon}^{\prime}\right)$ and $B(t) \varepsilon \&\left(W, W^{\prime}\right)$ such that

$$
\begin{array}{rlrl}
a_{\epsilon}(t ; u, v) & =\left(\Theta_{\epsilon}(t) u, v\right), & u, v \varepsilon V_{\epsilon}, \\
b_{\epsilon}(t ; u, v) & =\left(\oiint_{\epsilon}(t) u, v\right), & & u, v \varepsilon W_{\epsilon}, \\
b(t ; u, v) & =\left(\oiint_{(t) u, v),}\right. & u, v \varepsilon W .
\end{array}
$$

We consider the two problems

$$
\begin{gathered}
a_{\epsilon}(t) u_{\epsilon}^{\prime \prime}+B_{\epsilon}(t) u_{\epsilon}=f_{\epsilon}, \quad 0<t<T \\
u_{\epsilon}(0)=x_{\epsilon}, \quad u_{\epsilon}^{\prime}(0)=y_{\epsilon}
\end{gathered}
$$


and

$$
\begin{gathered}
u^{\prime \prime}+\beta(t) u=f, \quad 0<t<T, \\
u(0)=x, \quad u^{\prime}(0)=y
\end{gathered}
$$

where $^{\prime}=d / d t$ in (1.2) and (1.3). The purpose of this paper is to estimate the rate of convergence of $u_{\epsilon}$ to $u$, assuming $Q_{\epsilon}(t) \rightarrow I$ (identify on $W^{\prime}$ ), $\bigotimes_{\epsilon}(t) \rightarrow B(t)$, $f_{.} \rightarrow f, x_{\epsilon} \rightarrow x$ and $y_{\epsilon} \rightarrow y$ in some appropriate fashion.

(1.2) is among a class of equations called Sobolev type equations after S. L. Sobolev who studied the equation

$$
\left(\frac{\partial^{2}}{\partial x^{2}}+\frac{\partial^{2}}{\partial y^{2}}+\frac{\partial^{2}}{\partial z^{2}}\right) \frac{\partial^{2} u}{\partial t^{2}}+\frac{\partial^{2} u}{\partial z^{2}}=0
$$

in connection with a problem arising in the theory of fluids [12]. In general, Sobolev equations refer to partial differential equations in which mixed spacetime derivatives occur in the terms of highest order. For Sobolev equations involving only a first order time derivative (sometimes called pseudoparabolic equations) a considerable body of theory has evolved within the last several years (for singular perturbation problems involving such equations see $[7,8]$ ), but for Sobolev equations with higher order time derivatives many open problems still remain.

The next section contains preliminary material while our principal results for the problems (1.2), (1.3) are given in section 3. In order to derive the convergence estimates, we shall make use of some aspects of the theory of intermediate spaces. There are a number of papers which also employ interpolation theory to derive estimates somewhat related to those given here, in particular $[2,3,4,6]$. In section 4 the results of section 3 are applied to singular perturbations in a class of hyperbolic boundary value problems.

2. Preliminaries. The forms $a_{\epsilon}(t ; u, v), b_{\epsilon}(t ; u, v)$ and $b(t ; u, v)$ are assumed to satisfy the following (in order to simplify measurability considerations, we suppose $V_{\mathrm{e}}$ is separable, although this is not essential):

(2.1) For $u, v \varepsilon V_{\epsilon}$ we have $a_{\epsilon}(t ; u, v)=\overline{a_{\epsilon}(t ; v, u)}$; the map $t \rightarrow a_{\epsilon}(t ; u, v)$ is differentiable, the derivative $a_{\epsilon}{ }^{\prime}(t ; u, v)$ is measurable on $[0, T]$ and

$$
\begin{gathered}
\left|a_{\epsilon}(t ; u, v)\right|+\left|a_{\epsilon}{ }^{\prime}(t ; u, v)\right| \leqq M_{\epsilon}|u|_{V_{\epsilon}}|v|_{V_{\epsilon}}+M|u||v|, \\
a_{\epsilon}(t ; v, v) \geqq \alpha_{\epsilon}|v|_{V_{e}}{ }^{2}+\alpha|v|^{2}
\end{gathered}
$$

where $\alpha_{\epsilon}, \alpha, M_{\epsilon}$ and $M$ are positive constants.

(2.2) For $u, v \varepsilon W_{\epsilon}$ we have $b_{\epsilon}(t ; u, v)=\overline{b_{\epsilon}(t ; v, u)}$; the map $t \rightarrow b_{\epsilon}(t ; u, v)$ is differentiable, the derivative $b_{\epsilon}{ }^{\prime}(t ; u, v)$ is measurable on $[0, T]$ and

$$
\begin{gathered}
\left|b_{\epsilon}(t ; u, v)\right|+\left|b_{\epsilon}{ }^{\prime}(t ; u, v)\right| \leqq N_{\epsilon}|u|_{W_{\epsilon}}|v|_{W_{\epsilon}}+N|u|_{W}|v|_{W}, \\
b_{\epsilon}(t ; v, v) \geqq \beta_{\epsilon}|v|_{W_{\epsilon}}{ }^{2}+\beta|v|_{W}{ }^{2}
\end{gathered}
$$


where $\beta_{\epsilon}, \beta, N_{\epsilon}$ and $N$ are positive constants.

(2.3) For $u, v \varepsilon W$ we have $b(t ; u, v)=\overline{b(t ; v, u)}$; the map $t \rightarrow b(t ; u, v)$ is differentiable, the derivative $b^{\prime}(t ; u, v)$ is measurable on $[0, T]$ and

$$
|b(t ; u, v)|+\left|b^{\prime}(t ; u, v)\right| \leqq K|u|_{W}|v|_{W}, \quad b(t ; v, v) \geqq \gamma|v|_{W}{ }^{2}
$$

with positive constants $\gamma$ and $K$.

The form $a_{\epsilon}(t ; u, v)$ is compared with $(u, v)$ and $b_{\epsilon}(t ; u, v)$ compared with $b(t ; u, v)$ in the following way.

(2.4) Set

$$
\begin{aligned}
& r_{\epsilon}(t ; u, v)=a_{\epsilon}(t ; u, v)-(u, v), \quad u, v \varepsilon V_{\epsilon}, \\
& s_{\epsilon}(t ; u, v)=b_{\epsilon}(t ; u, v)-b(t ; u, v), \quad u, v \varepsilon W_{\epsilon} .
\end{aligned}
$$

We assume

$$
\begin{aligned}
& \left|r_{\epsilon}(t ; u, v)\right|+\left|r_{\epsilon}{ }^{\prime}(t ; u, v)\right| \leqq \rho_{\epsilon}|u|_{V_{\mathrm{e}}}|v|_{V_{\mathrm{\epsilon}}} \\
& \left|s_{\epsilon}(t ; u, v)\right|+\left|s_{\epsilon}{ }^{\prime}(t ; u, v)\right| \leqq \sigma_{\epsilon}|u|_{W_{e}}|v|_{W_{\mathrm{e}}}
\end{aligned}
$$

where

$$
\frac{\rho_{\epsilon}}{\alpha_{\epsilon}}=\mathrm{O}(1), \quad \frac{\sigma_{\epsilon}}{\beta_{\epsilon}}=\mathrm{O}(1) \quad \text { as } \quad \epsilon \rightarrow 0_{+} .
$$

It follows from (2.4) that we may suppose without loss of generality that

$$
\frac{M_{\epsilon}}{\alpha_{\epsilon}}=\mathrm{O}(1), \quad \frac{N_{\epsilon}}{\beta_{\epsilon}}=\mathrm{O}(1) \quad \text { as } \quad \epsilon \rightarrow 0_{+} .
$$

A few ideas concerning particular intermediate spaces of J. Peetre will be needed in the next section (see [1, Chapter 3] for details). Let $Y$ and $Z$ be Banach spaces, both of which are contained algebraically and topologically in some locally convex topological vector space, and let $Y+Z$ denote their algebraic sum. $Y+Z$ is a Banach space under the norm

$$
|x|_{Y+Z}=\inf _{x=y+z}\left(|y|_{Y}+|z|_{Z}\right), \quad y \varepsilon Y, \quad z \varepsilon Z .
$$

For each $x \varepsilon Y+Z$ and $t>0$ set

$$
K(t ; x)=\inf _{x=y+z}\left(|y|_{Y}+t|z|_{Z}\right), \quad y \varepsilon Y, \quad z \varepsilon Z .
$$

If $0<\theta<1$, the intermediate space $[Y, Z]_{\theta}$ is defined as follows:

$$
[Y, Z]_{\theta}=\left\{x: x \varepsilon Y+Z, t^{-\theta-1 / 2} K(t ; x) \varepsilon L^{2}(0, \infty)\right\} .
$$

It is a Banach space under the norm

$$
|x|_{[Y, Z]_{\theta}}=\left[\int_{0}^{\infty}\left(t^{-\theta-1 / 2} K(t ; x)\right)^{2} d t\right]^{1 / 2}
$$


The spaces $[Y, Z]_{\theta}$ have the interpolation property: let $\tilde{Y}, \widetilde{Z}$ be a second pair of Banach spaces with properties analogous to those of $Y, Z$, and let $R \varepsilon \mathcal{L}(Y+Z$, $\tilde{Y}+\widetilde{Z})$ be such that $R_{Y} \varepsilon \mathfrak{L}(Y, \tilde{Y})$ and $R_{Z} \varepsilon \mathcal{L}(Z, \widetilde{Z})$ where the subscripts indicate restrictions. Let

$$
M_{0}=\left\|R_{Y}\right\|_{L(Y, \tilde{Y})}, \quad M_{1}=\left\|R_{Z}\right\|_{L(Z, \tilde{Z})} .
$$

Then the following is true.

Proposition 2.1. The restriction of $R$ to $[Y, Z]_{\theta}$ is a bounded linear mapping of $[Y, Z]_{\theta}$ into $[\tilde{Y}, \widetilde{Z}]_{\theta}$ and the operator norm $M_{\theta}$ of this restriction satisfies

$$
M_{\theta} \leqq M_{0}{ }^{1-\theta} M_{1}{ }^{\theta} \text {. }
$$

Suppose now that $Y$ and $Z$ are Hilbert spaces with $Y \subset Z$ algebraically and topologically such that $Y$ is dense in $Z$. Then $[Y, Z]_{\theta}$ coincides (with equivalent norm) with the intermediate space by quadratic interpolation between $Y$ and $Z$ of index $\theta$ introduced by Lions ([9]; c.f. [10, Chapter 1]). For $k$ a nonnegative integer set

$$
H^{k}(0, T ; Y)=\left\{u: u, u^{\prime}, \cdots, u^{(k)} \varepsilon L^{2}(0, T ; Y)\right\} .
$$

It is a Hilbert space under the norm

$$
|u|_{H^{k}(0, T ; Y)}=\left[\int_{0}^{T} \sum_{i=0}^{k}\left|u^{(j)}(t)\right|_{Y}^{2} d t\right]^{1 / 2}
$$

$H^{k}(0, T ; X)$ is defined analogously.

Proposition 2.2. For $0<\theta<1,\left[H^{k}(0, T ; Y), H^{k}(0, T ; Z)\right]_{\theta}=$ $H^{k}\left(0, T ;[Y, Z]_{\theta}\right)$.

The proof of this proposition can proceed exactly as does the proof of Theorem 1.14 .2 of [10].

3. Rate of Convergence. We first make precise the problems (1.2) and (1.3). The precise form of (1.2) is

$$
\left\{\begin{array}{l}
a_{\epsilon}(t) u_{\epsilon}^{\prime \prime}+\beta_{\epsilon}(t) u_{\epsilon}=f_{\epsilon} \varepsilon L^{2}\left(0, T ; V_{\epsilon}^{\prime}\right), \quad 0<t<T, \\
u_{\epsilon}(0)=x_{\epsilon} \varepsilon V_{\epsilon}, \quad u_{\epsilon}^{\prime}(0)=y_{\epsilon} \varepsilon V_{\epsilon}, \\
u_{\epsilon} \varepsilon H^{2}\left(0, T ; V_{\epsilon}\right) .
\end{array}\right.
$$

Problem (1.3) is

$$
\left\{\begin{array}{l}
u^{\prime \prime}+B(t) u=f \varepsilon L^{2}(0, T ; X), \quad 0<t<T, \\
u(0)=x \varepsilon W, \quad u^{\prime}(0)=y \varepsilon X, \\
u \varepsilon L^{\infty}(0, T ; W), \quad u^{\prime} \varepsilon L^{\infty}(0, T ; X) .
\end{array}\right.
$$

The unique soluability of (3.2) is proved in [10, Theorem 3.8.1]. A general existence-uniqueness theorem for a class of problems which contains (3.1) is proved in the Appendix. 
For the remainder of this section, we shall assume that the data in (3.1) and (3.2) satisfy the additional conditions

$$
f_{\varepsilon} \varepsilon L^{2}(0, T ; X), \quad y \varepsilon W .
$$

The following theorem describes the behavior of $u_{\epsilon}-u$ as $\epsilon \rightarrow 0_{+}$.

Theorem 3.1. Assume (2.1)-(2.4), (3.3) and that the mapping $t \rightarrow u(t)-$ $x-t y \equiv v(t)$ belongs to $\left[Y_{\epsilon}, Z\right]_{\theta} \equiv\left[H^{2}\left(0, T ; V_{\epsilon}\right), H^{2}(0, T ; X) \cap H^{1}(0, T ; W)\right]_{\theta}$ for some $\theta \varepsilon(0,1)$. Then

$$
\begin{aligned}
\left|u_{\epsilon}^{\prime}-u^{\prime}\right|_{L^{\infty}(0, T ; X)}+\left|u_{\epsilon}-u\right|_{L^{\infty}(0, T ; W)} & \leqq C\left\{\left[\max \left(\frac{\rho_{\epsilon}}{\alpha_{\epsilon}^{1 / 2}}, \frac{\sigma_{\epsilon}}{\beta_{\epsilon}{ }^{1 / 2}}\right)\right]^{1-\theta} \cdot|v|_{L_{\left.Y_{\epsilon}, Z\right]_{\theta}}}\right. \\
& +\left|f_{\epsilon}-f\right|_{L^{2}(0, T ; X)}+\left|x_{\epsilon}-x\right|_{W} \\
& \left.+\left|y_{\epsilon}-y\right|_{W}+\frac{\sigma_{\epsilon}}{\beta_{\epsilon}^{1 / 2}}\left(\left|x_{\epsilon}\right|_{W \cdot}+\left|y_{\epsilon}\right|_{W_{\epsilon}}\right)\right\}
\end{aligned}
$$

where $C$ does not depend on $\epsilon, u$ or $\theta$.

Remark. $H^{2}(0, T ; X) \cap H^{1}(0, T ; W)$ is the Hilbert space $\left\{u: u, u^{\prime} \varepsilon\right.$ $\left.L^{2}(0, T ; W), u^{\prime \prime} \varepsilon L^{2}(0, T ; X)\right\}$ with the norm defined by

$$
\left.\left(|u|_{H^{1}(0, T ; W)}^{2}+\mid{u^{\prime \prime}}_{L^{2}(0, T ; X)}\right)^{2}\right)^{1 / 2} .
$$

The regularity hypothesis on $u$ is difficult to verify in practice because of the problem of finding a "concrete" characterization of $\left[Y_{\epsilon}, Z\right]_{\theta}$. In this respect the following corollary is more satisfactory.

Corollary 3.1. Assume (2.1)-(2.4), (3.3) and that $u \varepsilon H^{2}\left(0, T ;\left[V_{\epsilon}, W\right]_{\theta}\right)$ for some $\theta \varepsilon(0,1)$. Then $(3.4)$ holds with the term $|v|_{\left[Y_{\varepsilon}, Z\right]_{\theta}}$ replaced by

$$
|u|_{H^{2}\left(0, T ;\left[V_{e}, W\right] \theta\right)}+|x|_{\left[V_{e}, W\right] \theta}+|y|_{\left[V_{e}, W\right]_{\theta}} .
$$

Proof. Since $H^{2}(0, T ; W) \subset H^{2}(0, T ; X) \cap H^{1}(0, T ; W)$ with continuous injection we have, in view of Proposition 2.2,

$$
H^{2}\left(0, T ;\left[V_{\epsilon}, W\right]_{\theta}\right) \subset\left[H^{2}\left(0, T ; V_{\epsilon}\right), H^{2}(0, T ; X) \cap H^{1}(0, T ; W)\right]_{\theta}
$$

with continuous injection. The corollary follows from (3.5) and Theorem 3.1.

Proof of Theorem 3.1. We first reduce the problem of estimating $u_{\epsilon}-u$ to one in which $f_{\epsilon}=f$ and $x_{\epsilon}=y_{\epsilon}=x=y=0$. To do this we set

$$
\tilde{u}_{\epsilon}=u_{\epsilon}-x_{\epsilon}-t y_{\epsilon}, v=u-x-t y .
$$

Then $\tilde{u}_{\epsilon}$ satisfies

$$
\begin{gathered}
Q_{\epsilon}(t) \tilde{u}_{\epsilon}^{\prime \prime}+B_{\epsilon}(t) \tilde{u}_{\epsilon}=f_{\epsilon}(t)-\bigotimes_{\epsilon}(t)\left(x_{\epsilon}+t y_{\epsilon}\right), \\
\tilde{u}_{\epsilon} \varepsilon H^{2}\left(0, T ; V_{\epsilon}\right), \quad \tilde{u}_{\epsilon}(0)=\tilde{u}_{\epsilon}{ }^{\prime}(0)=0,
\end{gathered}
$$


and $v$ satisfies

$$
\begin{gathered}
v^{\prime \prime}+B(t) v=f(t)-B(t)(x+t y), \\
\text { v } \varepsilon L^{\infty}(0, T ; W), \quad v^{\prime} \varepsilon L^{\infty}(0, T ; X), \quad v(0)=v^{\prime}(0)=0 .
\end{gathered}
$$

Note that, by hypothesis, $v \varepsilon\left[Y_{\epsilon}, Z\right]_{\theta}$.

Let $v_{\epsilon}$ be the unique solution of

$$
\begin{gathered}
Q_{\epsilon}(t) v_{\epsilon}^{\prime \prime}+\beta_{\epsilon}(t) v_{\epsilon}=f(t)-\beta(t)(x+t y), \\
v_{\epsilon} \varepsilon H^{2}\left(0, T ; V_{\epsilon}\right), \quad v_{\epsilon}(0)=v_{\epsilon}^{\prime}(0)=0 .
\end{gathered}
$$

Then

$$
u_{\epsilon}-u=\left(\tilde{u}_{\epsilon}-v_{\epsilon}\right)+\left(v_{\epsilon}-v\right)+\left(x_{\epsilon}-x\right)+t\left(y_{\epsilon}-y\right)
$$

so that it suffices to estimate $\tilde{u}_{\epsilon}-v_{\epsilon}$ and $v_{\epsilon}-v$.

We proceed to estimate $\tilde{u}_{\epsilon}-v_{\epsilon} \equiv w_{\epsilon}$. For every $\xi \varepsilon V_{\epsilon}$ and almost all $t \varepsilon[0, T]$

$$
\begin{gathered}
a_{\epsilon}\left(t ; w_{\epsilon}^{\prime \prime}, \xi\right)+b_{\epsilon}\left(t ; w_{\epsilon}, \xi\right)=\left(f_{\epsilon}(t)-f(t), \xi\right) \\
-s_{\epsilon}\left(t ; x_{\epsilon}+t y_{\epsilon}, \xi\right)-b\left(t ; x_{\epsilon}-x, \xi\right)-t b\left(t ; y_{\epsilon}-y, \xi\right), \\
w_{\epsilon} \varepsilon H^{2}\left(0, T ; V_{\epsilon}\right), \quad w_{\epsilon}(0)=w_{\epsilon}^{\prime}(0)=0 .
\end{gathered}
$$

Let $\xi=w_{\epsilon}^{\prime}(t)$, take real parts and integrate from 0 to $t$. Using (2.1)-(2.4) we obtain

$$
\begin{aligned}
\frac{\alpha_{\epsilon}}{2}\left|w_{\epsilon}{ }^{\prime}(t)\right|_{V^{\prime}}{ }^{2}+ & \frac{\alpha}{2}\left|w_{\epsilon}{ }^{\prime}(t)\right|^{2}+\frac{\beta_{\epsilon}}{2}\left|w_{\epsilon}(t)\right|_{W_{\epsilon}}{ }^{2} \\
& +\frac{\beta}{2}\left|w_{\epsilon}(t)\right|_{W}{ }^{2} \leqq \int_{0}^{t}\left[\left(f_{\epsilon}(s)-f(s), w_{\epsilon}{ }^{\prime}(s)\right)\right. \\
& +\frac{1}{2} a_{\epsilon}{ }^{\prime}\left(s ; w_{\epsilon}{ }^{\prime}(s), w_{\epsilon}{ }^{\prime}(s)\right)+\frac{1}{2} b_{\epsilon}{ }^{\prime}\left(s ; w_{\epsilon}(s), w_{\epsilon}(s)\right) \\
& -\operatorname{Re} s_{\epsilon}\left(s ; x_{\epsilon}+s y_{\epsilon}, w_{\epsilon}{ }^{\prime}(s)\right)-\operatorname{Re} b\left(s ; x_{\epsilon}-x, w_{\epsilon}{ }^{\prime}(s)\right) \\
& \left.-\operatorname{Re} b\left(s ; s\left(y_{\epsilon}-y\right), w_{\epsilon}{ }^{\prime}(s)\right)\right] d s .
\end{aligned}
$$

In what follows we shall set, for example,

$$
\begin{gathered}
v_{\epsilon}(t)=L^{2}\left(0, t ; V_{\epsilon}\right), \quad V_{\epsilon}^{\prime}(t)=L^{2}\left(0, t ; V_{\epsilon}^{\prime}\right), \\
v_{\epsilon}=v_{\epsilon}(T), \quad V_{\epsilon}^{\prime}=V_{\epsilon}^{\prime}(T) .
\end{gathered}
$$

Other spaces $W_{\epsilon}(t), W_{\epsilon}, W_{(t)}, W, x(t), x$, etc., are defined analogously. Also, $C$ will denote a generic constant independent of $u$ and $\epsilon$.

Returning to (3.6), after an integration by parts in the last three terms we obtain the following upper bound on the right side of (3.6): 


$$
\begin{aligned}
& C\left[\left|f_{\epsilon}-f\right|_{\mathscr{X}}\left|w_{\epsilon}{ }^{\prime}\right|_{\mathscr{X}(t)}+M_{\epsilon}\left|w_{\epsilon}{ }^{\prime}\right|_{\mathcal{V}_{\epsilon}(t)}{ }^{2}+\left|w_{\epsilon}{ }^{\prime}\right|_{\mathscr{X}(t)}{ }^{2}\right. \\
& +N_{\epsilon}\left|w_{\epsilon}\right|_{w_{\epsilon}(t)}{ }^{2}+\left|w_{\epsilon}\right|_{W_{(t)}}{ }^{2}+\sigma_{\epsilon}\left(\left|x_{\epsilon}\right|_{W_{\epsilon}}+\left|y_{\epsilon}\right|_{W_{\epsilon}}\right) \\
& \cdot\left(\left|w_{\epsilon}(t)\right|_{W_{\epsilon}}+\left|w_{\epsilon}\right|_{w_{e}(t)}\right) \\
& \left.+\left(\left|x_{\epsilon}-x\right|_{W}+\left|y_{\epsilon}-y\right|_{W}\right) \cdot\left(\left|w_{\epsilon}(t)\right|_{W}+\left|w_{\epsilon}\right|_{W(t)}\right)\right] \text {. }
\end{aligned}
$$

Using the inequality $2 a b \leqq \delta a^{2}+(1 / \delta) b^{2}(\delta>0)$ we can obtain from (2.5), (3.6) and (3.7) the estimate

$\alpha_{\epsilon}\left|w_{\epsilon}{ }^{\prime}(t)\right|_{V_{\epsilon}}{ }^{2}+\left|w_{\epsilon}{ }^{\prime}(t)\right|^{2}+\beta_{\epsilon}\left|w_{\epsilon}(t)\right|_{W_{e}}{ }^{2}+\left|w_{\epsilon}(t)\right|_{W}{ }^{2}$

$$
\begin{aligned}
& \leqq C\left[\alpha_{\epsilon}\left|w_{\epsilon}\right|_{V_{\epsilon}(t)}{ }^{2}+\left|w_{\epsilon}{ }^{\prime}\right|_{\mathscr{X}(t)}{ }^{2}+\beta_{\epsilon}\left|w_{\epsilon}\right|_{W_{\epsilon}(t)}{ }^{2}\right. \\
& +\left|w_{\epsilon}\right|_{W(t)}{ }^{2}+\left|f_{\epsilon}-f\right|_{X^{2}}{ }^{2}+\frac{\sigma_{\epsilon}{ }^{2}}{\beta_{\epsilon}}\left(\left|x_{\epsilon}\right|_{W_{\epsilon}}+\left|y_{\epsilon}\right|_{W_{\epsilon}}\right)^{2} \\
& \left.+\left(\left|x_{\epsilon}-x\right|_{W}+\left|y_{\epsilon}-y\right|_{W}\right)^{2}\right] .
\end{aligned}
$$

An application of Gronwall's lemma yields

$\alpha_{\epsilon}\left|w_{\epsilon}{ }^{\prime}(t)\right|_{V_{\epsilon}}{ }^{2}+\left|w_{\epsilon}{ }^{\prime}(t)\right|^{2}+\beta_{\epsilon}\left|w_{\epsilon}(t)\right|_{W \cdot}{ }^{2}+\left|w_{\epsilon}(t)\right|_{W}{ }^{2}$

$$
\leqq C\left[\left|f_{\epsilon}-f\right|_{x^{2}}{ }^{2}+\left(\left|x_{\epsilon}-x\right|_{W}+\left|y_{\epsilon}-y\right|_{W}\right)^{2}+\frac{\sigma_{\epsilon}{ }^{2}}{\beta_{\epsilon}}\left(\left|x_{\epsilon}\right|_{W \cdot}+\left|y_{\epsilon}\right|_{W}\right)^{2}\right] \text {. }
$$

We therefore deduce the estimate

$$
\begin{aligned}
& \left|w_{\epsilon}^{\prime}\right|_{L^{\infty}(0, T ; X)}+\left|w_{\epsilon}\right|_{L^{\infty}(0, T ; W)} \\
& \quad \leqq C\left[\left|f_{\epsilon}-f\right|_{L^{2}(0, T ; X)}+\left|x_{\epsilon}-x\right|_{W}+\left|y_{\epsilon}-y\right|_{W}+\frac{\sigma_{\epsilon}}{\beta_{\epsilon}{ }^{1 / 2}}\left(\left|x_{\epsilon}\right|_{W \epsilon}+\left|y_{\epsilon}\right|_{W \epsilon}\right)\right] .
\end{aligned}
$$

Since

$$
u_{\epsilon}-u=w_{\epsilon}+\left(v_{\epsilon}-v\right)+\left(x_{\epsilon}-x\right)+t\left(y_{\epsilon}-y\right),
$$

to complete the proof of Theorem 3.1 we must show that

$$
\begin{aligned}
\left|v_{\epsilon}^{\prime}-v^{\prime}\right|_{L^{\infty}(0, T ; X)}+\left|v_{\epsilon}-v\right|_{L^{\infty}(0, T ; W)} & \\
& \leqq C\left[\max \left(\frac{\rho_{\epsilon}}{\alpha_{\epsilon}{ }^{1 / 2}}, \frac{\sigma_{\epsilon}}{\beta_{\epsilon}{ }^{1 / 2}}\right)\right]^{1-\theta}|v|_{\left[Y_{\epsilon}, Z\right]_{\theta}} .
\end{aligned}
$$

To prove (3.8), it suffices to prove the following two special cases:

$$
\text { If } v \varepsilon Y_{\epsilon}=H^{2}\left(0, T ; V_{\epsilon}\right) \text {, then } \begin{aligned}
\mid v_{\epsilon}^{\prime} & -\left.v^{\prime}\right|_{L^{\infty}(0, T ; X)}+\left|v_{\epsilon}-v\right|_{L^{\infty}(0, T ; W)} \\
& \leqq C \max \left(\frac{\rho_{\epsilon}}{\alpha_{\epsilon}^{1{ }^{1 / 2}}}, \frac{\sigma_{\epsilon}}{\beta_{\epsilon}^{1 / 2}}\right)|v|_{H^{2}\left(0, T ; V_{\mathrm{e}}\right)} .
\end{aligned}
$$

If $v \varepsilon Z=H^{2}(0, T ; X) \cap H^{1}(0, T ; W)$, then

$$
\left|v_{\epsilon}^{\prime}-v^{\prime}\right|_{L^{\infty}(0, T ; X)}+\left|v_{\epsilon}-v\right|_{L^{\infty}(0, T ; W)} \leqq C|v|_{Z} .
$$


In fact, assuming (3.9) and (3.10) to hold, let $v$ be given in $H^{2}(0, T ; X) \cap$ $H^{1}(0, T ; W)$ (for example) with $v(0)=v^{\prime}(0)=0$, and let $v_{\epsilon}$ be the unique solution in $H^{2}\left(0, T ; V_{\epsilon}\right)$ of

$$
Q_{\epsilon}(t) v_{\epsilon}^{\prime \prime}+B_{\epsilon}(t) v_{\epsilon}=v^{\prime \prime}+B_{B}(t) v, \quad v_{\epsilon}(0)=v_{\epsilon}^{\prime}(0)=0 .
$$

A linear mapping $R_{\epsilon}$ is then defined by setting

Consider the Banach space

$$
R_{\epsilon} v=v_{\epsilon}-v
$$

$$
H_{\infty}{ }^{1}(0, T ; W, X)=\left\{u: u \varepsilon L^{\infty}(0, T ; W), \quad u^{\prime} \varepsilon L^{\infty}(0, T ; X)\right\}
$$

with the norm defined by

$$
|u|_{L^{\infty}(0, T ; W)}+\left|u^{\prime}\right|_{L^{\infty}(0, T ; X)} .
$$

(3.9) and (3.10) show that $R_{\epsilon}$ is bounded both as a mapping on $Z=H^{2}(0, T ; X)$ $\cap H^{1}(0, T ; W)$ and as a mapping on $Y_{\epsilon}=H^{2}\left(0, T ; V_{\epsilon}\right)$ into $H_{\infty}{ }^{1}(0, T ; W, X)$. It follows from Proposition 2.1 that the restriction of $R_{\epsilon}$ to $\left[Y_{\epsilon}, Z\right]_{\theta}$ is bounded as a mapping from this space into $H_{\infty}{ }^{1}(0, T ; W, X)$ with bound

$$
M_{\theta} \leqq C\left[\max \left(\frac{\rho_{\epsilon}}{\alpha_{\epsilon}{ }^{1 / 2}}, \frac{\sigma_{\epsilon}}{\beta_{\epsilon}{ }^{1 / 2}}\right)\right]^{1-\theta}
$$

where $C$ does not depend on $\epsilon$ or $\theta$. The last estimate is exactly the content of (3.8).

We proceed to the proof of (3.9). From (3.1) and (3.2) we obtain the following equality, valid for almost all $t$ in $[0, T]$ :

$$
\begin{aligned}
& a_{\epsilon}\left(t ; v_{\epsilon}^{\prime \prime}-v^{\prime \prime}, v_{\epsilon}{ }^{\prime}-v^{\prime}\right)+b_{\epsilon}\left(t ; v_{\epsilon}-v, v_{\epsilon}^{\prime}-v^{\prime}\right) \\
&=-r_{\epsilon}\left(t ; v^{\prime \prime}, v_{\epsilon}^{\prime}-v^{\prime}\right)-s_{\epsilon}\left(t ; v, v_{\epsilon}^{\prime}-v^{\prime}\right) .
\end{aligned}
$$

We take the real part and integrate from 0 to $t$. After an integration by parts in the last term we obtain the estimate

$$
\begin{aligned}
\frac{\alpha_{\epsilon}}{2}\left|v_{\epsilon}{ }^{\prime}(t)-v^{\prime}(t)\right|_{V_{e}}{ }^{2}+\frac{\alpha}{2} \mid v_{\epsilon}{ }^{\prime}(t) & -\left.v^{\prime}(t)\right|^{2} \\
& +\frac{\beta_{\epsilon}}{2}\left|v_{\epsilon}(t)-v(t)\right|_{W_{\epsilon}}{ }^{2}+\frac{\beta}{2}\left|v_{\epsilon}(t)-v(t)\right|_{W}{ }^{2} \\
& \leqq \frac{M_{\epsilon}}{2}\left|v_{\epsilon}{ }^{\prime}-v^{\prime}\right|_{v_{\epsilon}(t)}{ }^{2}+\frac{M}{2}\left|v_{\epsilon}{ }^{\prime}-v^{\prime}\right|_{\mathcal{X}_{(t)}}{ }^{2} \\
& +\frac{N_{\epsilon}}{2}\left|v_{\epsilon}-v\right|_{W_{\epsilon}(t)}{ }^{2}+\frac{N}{2}\left|v_{\epsilon}-v\right|_{W_{(}(t)}{ }^{2} \\
& +\frac{\rho_{\epsilon}{ }^{2}}{2 \alpha_{\epsilon}}\left|v^{\prime \prime}\right|_{v_{\epsilon}(t)}{ }^{2}+\frac{\alpha_{\epsilon}}{2}\left|v_{\epsilon}{ }^{\prime}-v^{\prime}\right|_{v_{\epsilon}(t)}{ }^{2} \\
& +\frac{\sigma_{\epsilon}{ }^{2}}{\beta_{\epsilon}}|v(t)|_{W_{\epsilon}}{ }^{2}+\frac{\beta_{\epsilon}}{4}\left|v_{\epsilon}(t)-v(t)\right|_{W_{\epsilon}}{ }^{2} \\
& +\frac{\sigma_{\epsilon}{ }^{2}}{2 \beta_{\epsilon}}\left(|v|_{W_{\epsilon}(t)}{ }^{2}+\left|v^{\prime}\right|_{W_{\epsilon}(t)}{ }^{2}\right)+\beta_{\epsilon}\left|v_{\epsilon}-v\right|_{W_{\epsilon}(t)}{ }^{2} .
\end{aligned}
$$


Therefore

$$
\begin{aligned}
\alpha_{\epsilon} & \left|v_{\epsilon}{ }^{\prime}(t)-v^{\prime}(t)\right|_{V_{e}}{ }^{2}+\left|v_{\epsilon}{ }^{\prime}(t)-v^{\prime}(t)\right|^{2}+\beta_{\epsilon}\left|v_{\epsilon}(t)-v(t)\right|_{W e}{ }^{2} \\
+ & +\left|v_{\epsilon}(t)-v(t)\right|_{W}{ }^{2} \leqq C\left[\alpha_{\epsilon}\left|v_{\epsilon}{ }^{\prime}-v^{\prime}\right|_{v_{\epsilon}(t)}{ }^{2}+\left|v_{\epsilon}{ }^{\prime}-v^{\prime}\right|_{X(t)}{ }^{2}+\beta_{\epsilon}\left|v_{\epsilon}-v\right|_{W_{e}(t)}{ }^{2}\right. \\
& \left.+\left|v_{\epsilon}-v\right|_{W(t)}{ }^{2}+\frac{\rho_{\epsilon}{ }^{2}}{\alpha_{\epsilon}}\left|v^{\prime \prime}\right|_{v_{e}}{ }^{2}+\frac{{\sigma_{\epsilon}}^{2}}{\beta_{\epsilon}}\left(\left|v^{\prime}\right|_{W W_{\epsilon}}{ }^{2}+|v|_{W}{ }^{2}\right)\right]
\end{aligned}
$$

We have used the inequality

$$
|v(t)|_{w_{e}} \leqq T^{1 / 2}|v|_{w_{e}}
$$

in the last step. Applying Gronwall's lemma we obtain

$$
\begin{aligned}
\alpha_{\epsilon}\left|v_{\epsilon}{ }^{\prime}(t)-v^{\prime}(t)\right|_{v_{e}}{ }^{2}+\left|v_{\epsilon}{ }^{\prime}(t)-v^{\prime}(t)\right|^{2} & +\beta_{\epsilon}\left|v_{\epsilon}(t)-v(t)\right|_{W_{e}}{ }^{2}+\left|v_{\epsilon}(t)-v(t)\right|_{W}{ }^{2} \\
& \leqq C\left[\frac{\rho_{\epsilon}{ }^{2}}{\alpha_{\epsilon}}\left|v^{\prime \prime}\right|_{v_{\epsilon}}+\frac{\sigma_{\epsilon}{ }^{2}}{\beta_{\epsilon}}\left(\left|v^{\prime}\right|_{W_{e}}{ }^{2}+|v|_{W_{\epsilon}}{ }^{2}\right)\right]
\end{aligned}
$$

(3.9) follows from the last inequality.

To prove (3.10) we start with the equality

$$
\begin{aligned}
& \left(v_{\epsilon}^{\prime \prime}-v^{\prime \prime}, v_{\epsilon}^{\prime}-v^{\prime}\right)+b\left(t ; v_{\epsilon}-v, v_{\epsilon}^{\prime}-v^{\prime}\right) \\
& =-\left(v_{\epsilon}^{\prime \prime}-v^{\prime \prime}, v^{\prime}\right)-b\left(t ; v_{\epsilon}-v, v^{\prime}\right)-r_{\epsilon}\left(t ; v_{\epsilon}^{\prime \prime}, v_{\epsilon}^{\prime}\right)-s_{\epsilon}\left(t ; v_{\epsilon}, v_{\epsilon}^{\prime}\right) .
\end{aligned}
$$

Proceeding as before we obtain

$$
\begin{aligned}
\frac{1}{2} \mid v_{\epsilon}^{\prime}(t)- & \left.v^{\prime}(t)\right|^{2}+\frac{\gamma}{2}\left|v_{\epsilon}(t)-v(t)\right|_{W}^{2} \leqq \frac{K}{2}\left|v_{\epsilon}-v\right|_{\mathscr{W}(t)}^{2}+\left|v_{\epsilon}^{\prime}(t)-v^{\prime}(t)\right|\left|v^{\prime}(t)\right| \\
& +\left|v_{\epsilon}^{\prime}-v^{\prime}\right|_{\mathscr{X}(t)}\left|v^{\prime \prime}\right|_{\mathscr{X}(t)}+K\left|v_{\epsilon}-v\right|_{\mathscr{W}(t)}\left|v^{\prime}\right|_{\mathscr{W}(t)} \\
& -\frac{1}{2} r_{\epsilon}\left(t ; v_{\epsilon}^{\prime}(t), v_{\epsilon}^{\prime}(t)\right)+\frac{\rho_{\epsilon}}{2}\left|v_{\epsilon}^{\prime}\right|_{v_{\epsilon}(t)}{ }^{2}-\frac{1}{2} s_{\epsilon}\left(t ; v_{\epsilon}(t), v_{\epsilon}(t)\right)+\frac{\sigma_{\epsilon}}{2}\left|v_{\epsilon}\right|_{W_{\epsilon}(t)}{ }^{2}
\end{aligned}
$$

Therefore

$$
\begin{aligned}
& \left|v_{\epsilon}^{\prime}(t)-v^{\prime}(t)\right|^{2}+\left|v_{\epsilon}(t)-v(t)\right|_{W}{ }^{2} \leqq C\left[\left|v_{\epsilon}-v_{W(t)}{ }^{2}+\right| v_{\epsilon}{ }^{\prime}-\left.v^{\prime}\right|_{X(t)}{ }^{2}\right. \\
& \left.\quad+\left|v^{\prime \prime}\right|_{x}{ }^{2}+\left|v^{\prime}\right|_{W}{ }^{2}+\rho_{\epsilon}\left(\left|v_{\epsilon}{ }^{\prime}(t)\right|_{V_{\epsilon}}{ }^{2}+\left|v_{\epsilon}{ }^{\prime}\right|_{v_{\epsilon}(t)}{ }^{2}\right)+\sigma_{\epsilon}\left(\left|v_{\epsilon}(t)\right|_{W \epsilon}{ }^{2}+\left|v_{\epsilon}\right|_{W_{\epsilon}(t)}{ }^{2}\right)\right] .
\end{aligned}
$$

Applying Gronwall's lemma once again, we obtain

$$
\begin{aligned}
\left|v_{\epsilon}^{\prime}(t)-v^{\prime}(t)\right|^{2}+ & \left|v_{\epsilon}(t)-v(t)\right|_{W}{ }^{2} \leqq C\left[\left|v^{\prime \prime}\right|_{x^{2}}+\left|v^{\prime}\right|_{W}{ }^{2}+\rho_{\epsilon}\left|v_{\epsilon}^{\prime}\right|_{v_{\epsilon}(t)}{ }^{2}\right. \\
& \left.+\sigma_{\epsilon}\left|v_{\epsilon}\right|_{W_{\epsilon}(t)}{ }^{2}+\sup _{0 \leqq s \leqq t}\left(\rho_{\epsilon}\left|v_{\epsilon}^{\prime}(s)\right|_{V_{\epsilon}}{ }^{2}+\sigma_{\epsilon}\left|v_{\epsilon}(s)\right|_{W_{\epsilon}}{ }^{2}\right)\right] .
\end{aligned}
$$

We also have, for almost all $t$,

$$
a_{\epsilon}\left(t ; v_{\epsilon}{ }^{\prime \prime}, v_{\epsilon}{ }^{\prime}\right)+b_{\epsilon}\left(t ; v_{\epsilon}, v_{\epsilon}{ }^{\prime}\right)=\left(v^{\prime \prime}, v_{\epsilon}{ }^{\prime}\right)+b\left(t ; v, v_{\epsilon}{ }^{\prime}\right) \text {. }
$$

Taking real parts and integrating from 0 to $t$ we obtain, after an integration by parts in the second term on the right, 


$$
\begin{aligned}
\frac{\alpha_{\epsilon}}{2}\left|v_{\epsilon}{ }^{\prime}(t)\right|_{V_{e}}{ }^{2} & +\frac{\alpha}{2}\left|v_{\epsilon}{ }^{\prime}(t)\right|^{2}+\frac{\beta_{\epsilon}}{2}\left|v_{\epsilon}(t)\right|_{W e}{ }^{2}+\frac{\beta}{2}\left|v_{\epsilon}(t)\right|_{W}{ }^{2} \\
& \leqq \frac{M_{\epsilon}}{2}\left|v_{\epsilon}{ }^{\prime}\right|_{v_{\epsilon}(t)}{ }^{2}+\frac{M}{2}\left|v_{\epsilon}{ }^{\prime}\right|_{\mathscr{X}(t)}{ }^{2}+\frac{N_{\epsilon}}{2}\left|v_{\epsilon}\right|_{W_{e}(t)}{ }^{2}+\frac{N}{2}\left|v_{\epsilon}\right|_{W(t)}{ }^{2} \\
& +\left|v^{\prime \prime}\right|_{X(t)}\left|v_{\epsilon}{ }^{\prime}\right|_{X(t)}+C\left[|v(t)|_{W}\left|v_{\epsilon}(t)\right|_{W}+\left|v_{\epsilon}\right|_{W(t)}\left(|v|_{W(t)}+\left|v^{\prime}\right|_{W(t)}\right)\right] .
\end{aligned}
$$

From the last inequality we obtain

$$
\begin{aligned}
\alpha_{\epsilon}\left|v_{\epsilon}{ }^{\prime}(t)\right|_{V_{e}}{ }^{2}+\left|v_{\epsilon}{ }^{\prime}(t)\right|^{2}+\beta_{\epsilon}\left|v_{\epsilon}(t)\right|_{W \epsilon}{ }^{2} & +\left|v_{\epsilon}(t)\right|_{W}{ }^{2} \\
& \leqq C\left[\alpha_{\epsilon}\left|v_{\epsilon}{ }^{\prime}\right|_{v_{\epsilon}(t)}{ }^{2}+\left|v_{\epsilon}{ }^{\prime}\right|{ }_{X(t)}{ }^{2}+\beta_{\epsilon}\left|v_{\epsilon}\right|_{W_{\epsilon}(t)}{ }^{2}\right. \\
& \left.+\left|v_{\epsilon}\right|_{W(t)}{ }^{2}+\left|v^{\prime \prime}\right|_{x}{ }^{2}+\left|v^{\prime}\right|_{W}{ }^{2}+|v|_{W}{ }^{2}\right] .
\end{aligned}
$$

The use of Gronwall's lemma gives

$\alpha_{\epsilon}\left|v_{\epsilon}{ }^{\prime}(t)\right|_{v_{\epsilon}}{ }^{2}+\beta_{\epsilon}\left|v_{\epsilon}(t)\right|_{W_{\epsilon}}{ }^{2}+\left|v_{\epsilon}{ }^{\prime}(t)\right|^{2}+\left|v_{\epsilon}(t)\right|_{W}{ }^{2} \leqq C\left[\left|v^{\prime \prime}\right|_{x^{2}}{ }^{2}+\left|v^{\prime}\right|_{W}{ }^{2}+|v|_{W}{ }^{2}\right]$.

Using this last inequality in (3.11), together with (2.4), we obtain

$$
\begin{aligned}
\left|v_{\epsilon}^{\prime}-v^{\prime}\right|_{L^{\infty}(0, T ; X)}+ & \left|v_{\epsilon}-v\right|_{L^{\infty}(0, T ; W)} \\
& \leqq C\left[\left|v^{\prime \prime}\right|_{L^{2}(0, T ; X)}+\left|v^{\prime}\right|_{L^{2}(0, T ; W)}+|v|_{L^{2}(0, T ; W)}\right] \leqq C|v|_{Z} .
\end{aligned}
$$

4. Application. The following illustrates one kind of problem to which the results of the previous section can be applied.

Let $\Omega$ be a bounded open set in $R^{N}$ with boundary $\Gamma$. We shall be dealing with the Hilbert spaces (see [10, Chapter 1] for details)

$$
H^{m}(\Omega)=\left\{u: D^{i} u \varepsilon L^{2}(\Omega), \quad|i| \leqq m\right\}
$$

( $m=$ positive integer) and, by quadratic interpolation,

$$
H^{s}(\Omega)=\left[H^{m}(\Omega), L^{2}(\Omega)\right]_{\theta}, \quad(1-\theta) m=s>0 .
$$

The norm in $H^{s}(\Omega)$ is denoted by $|\cdot|_{s, \Omega} ;$ for $s=m$

$$
|u|_{m, \Omega}=\left[\int_{\Omega} \sum_{|i| \leqq m}\left|D^{i} u(x)\right|^{2} d x\right]^{1 / 2}
$$

$H_{0}{ }^{8}(\Omega)$ denotes the closure of $C_{0}{ }^{\infty}(\Omega)$ in $H^{s}(\Omega)$.

Let $\ell \leqq \ell^{\prime} \leqq m(\ell<m)$ be positive integers and $Q=\Omega \times(0, T)$. We consider the differential equation

$$
\begin{aligned}
& {[1+\alpha(\epsilon) A(x, t ; D)] \frac{\partial^{2} u_{\epsilon}}{\partial t^{2}}+[B(x, t ; D)+\beta(\epsilon) \widetilde{B}(x, t ; D)] u } \\
&=f_{\epsilon}(x, t), \quad(x, t) \varepsilon Q
\end{aligned}
$$

with the Dirichlet boundary conditions

$$
\left(\frac{\partial}{\partial n}\right)^{k} u_{\epsilon}=0, \quad(x, t) \varepsilon \Gamma \times(0, T), \quad k=0,1, \cdots, m-1
$$


and vanishing initial data

$$
u_{\epsilon}(x, 0)=0, \quad \frac{\partial u_{\epsilon}}{\partial t}(x, 0)=0, \quad x \varepsilon \Omega .
$$

$\alpha(\epsilon)$ and $\beta(\epsilon)$ are positive and converge to zero with $\epsilon$ and $A, B$ and $\widetilde{B}$ are differential operators in $x$ of respective orders $m, \ell$ and $\ell^{\prime}$, with coefficients defined in $\bar{Q}$, given by

$$
\begin{array}{ll}
A(x, t ; D)=\sum_{|i|,|j| \leqq m}(-1)^{i} D^{i}\left(a_{i j}(x, t) D^{i}\right), & a_{i j}=\overline{a_{i i}}, \\
B(x, t ; D)=\sum_{|i|,|j| \leqq \ell}(-1)^{i} D^{i}\left(b_{i j}(x, t) D^{j}\right), & b_{i j}=\overline{b_{i i}}, \\
\tilde{B}(x, t ; D)=\sum_{i|,| j \mid \leqq \ell^{\prime}}(-1)^{i} D^{i}\left(\tilde{b}_{i j}(x, t) D^{i}\right), & \tilde{b}_{i j}=\overline{\tilde{b}_{i i}} .
\end{array}
$$

We wish to compare the solution of $(4.1)_{\epsilon}-(4.3)_{\epsilon}$ with the solution of

$$
\begin{gathered}
\frac{\partial^{2} u}{\partial t^{2}}+B(x, t ; D) u=f(x, t), \quad(x, t) \varepsilon Q, \\
\left(\frac{\partial}{\partial n}\right)^{k} u=0, \quad(x, t) \varepsilon \Gamma \times(0, T), \quad k=0,1, \cdots, l-1, \\
u(x, 0)=0, \quad \frac{\partial u}{\partial t}(x, 0)=0, \quad x \varepsilon \Omega .
\end{gathered}
$$

Let $a(t ; u, v), b(t ; u, v)$ and $\tilde{b}(t ; u, v)$ be the sesquilinear forms on $H^{m}(\Omega), H^{\ell}(\Omega)$ and $H^{\ell^{\prime}}(\Omega)$, respectively, corresponding to $A, B$ and $\widetilde{B}$; for example

$$
a(t ; u, v)=\sum_{|i|,|i| \leqq m} \int_{\Omega} a_{i j} D^{i} u \overline{D^{i} v} d x, \quad u, v \varepsilon H^{m}(\Omega) .
$$

We assume the following

(4.4) $\Omega$ is a bounded open set in $R^{N}$ of class $C^{\infty}$.

$$
a_{i j}, \partial a_{i j} / \partial t, b_{i j}, \partial b_{i j} / \partial t, \tilde{b}_{i j} \quad \text { and } \quad \partial \tilde{b}_{i j} / \partial t \varepsilon L^{\infty}(\bar{Q}) .
$$

(4.6) The forms $a(t ; u, v), b(t ; u, v)$ and $\tilde{b}(t ; u, v)$ are elliptic on the spaces $H_{0}{ }^{m}(\Omega), H_{0}{ }^{\ell}(\Omega)$ and $H_{0}{ }^{\ell^{\prime}}(\Omega)$, respectively; thus

$$
\begin{aligned}
& a(t ; v, v) \geqq \tilde{\alpha}|v|_{m, \Omega}{ }^{2}, \quad v \varepsilon H_{0}{ }^{m}(\Omega), \quad \tilde{\alpha}>0, \\
& b(t ; v, v) \geqq \gamma|v|_{\ell, \Omega}{ }^{2}, \quad v \varepsilon H_{0}{ }^{\ell}(\Omega), \quad \gamma>0 \text {, } \\
& \tilde{b}(t ; v, v) \geqq \tilde{\beta}|v|_{\ell^{\prime}, \Omega}{ }^{2}, \quad v \varepsilon H_{0}^{\ell^{\prime}}(\Omega), \quad \tilde{\beta}>0 \text {. }
\end{aligned}
$$

If we now set

$$
\begin{aligned}
& a_{\epsilon}(t ; u, v)=(u, v)_{L^{2}(\Omega)}+\alpha(\epsilon) a(t ; u, v), \quad u, v \varepsilon H_{0}{ }^{m}(\Omega), \\
& b_{\epsilon}(t ; u, v)=b(t ; u, v)+\beta(\epsilon) \tilde{b}(t ; u, v), \quad u, v \varepsilon H_{0}^{\ell^{\prime}}(\Omega),
\end{aligned}
$$


then one easily verifies that the forms $a_{\epsilon}, b_{\epsilon}$ and $b$ satisfy (2.1)-(2.4) with respect to the spaces

$$
V_{\epsilon} \equiv H_{0}{ }^{m}(\Omega), \quad W_{\epsilon} \equiv H_{0}{ }^{{ }^{\prime}}(\Omega), \quad W=H_{0}{ }^{\ell}(\Omega) .
$$

The quantities $\alpha_{\epsilon}, M_{\epsilon}$ and $\rho_{\epsilon}$ (respectively, $\beta_{\epsilon}, N_{\epsilon}$ and $\sigma_{\epsilon}$ ) are constant multiples of $\alpha(\epsilon)$ (respectively, $\beta(\epsilon)$ ). It follows that $(4.1)_{\epsilon}-(4.3)_{\epsilon}(\epsilon>0)$ and $(4.1)_{0}-(4.3)_{0}$ have unique solutions $u_{\epsilon}$ and $u$, respectively, such that

$$
u_{\epsilon} \varepsilon H^{2}\left(0, T ; H_{0}{ }^{m}(\Omega)\right), \quad u \varepsilon L^{\infty}\left(0, T ; H_{0}{ }^{\ell}(\Omega)\right), \quad \frac{\partial u}{\partial t} \varepsilon L^{\infty}\left(0, T ; L^{2}(\Omega)\right)
$$

provided

$$
f \text { and } f_{\epsilon} \varepsilon L^{2}(Q) .
$$

Let $r$ and $s$ be nonnegative numbers. As in [10, Chapter 4] we define

$$
H^{r, s}(Q)=L^{2}\left(0, T ; H^{r}(\Omega)\right) \cap H^{s}\left(0, T ; L^{2}(\Omega)\right)
$$

which is a Hilbert space with norm

$$
\left[\int_{0}^{T}|u(t)|_{r, \Omega^{2}} d t+|u|_{H^{s}\left(0, T ; L^{2}(\Omega)\right)^{2}}\right]^{1 / 2}
$$

The space $H^{s}\left(0, T ; L^{2}(\Omega)\right)$ for $s \neq$ integer is defined by quadratic interpolation:

$$
H^{s}\left(0, T ; L^{2}(\Omega)\right)=\left[H^{m}\left(0, T ; L^{2}(\Omega)\right), L^{2}\left(0, T ; L^{2}(\Omega)\right)\right]_{\theta}
$$

where $m>s$ is a positive integer and $(1-\theta) m=s>0$. In order that $u$ satisfy the regularity hypothesis of Corollary 3.1 we assume that the data in $(4.1)_{0}-$ $(4.3)_{0}$ satisfy the following:

(4.8) Let $r$ be a positive number such that $r \geqq 1+1 / 4 \ell$ and $2 r \neq$ integer $+1 / 2$. Then

$$
\begin{gathered}
\left(\frac{\partial}{\partial t}\right)^{n} b_{i j} \varepsilon L^{\infty}(Q) \text { for } 0 \leqq n<2 r+1 / 2, \\
f \varepsilon H^{(2 r-1) \ell, 2 r}(Q), \quad f^{(n)}(0)=0 \text { for } 0 \leqq n<2 r-1 / 2 .
\end{gathered}
$$

It then follows from Theorem 5.8.1 of [10] that

$$
u \varepsilon H^{(2 r+1) \ell, 2 r+1}(Q) .
$$

In particular (c.f. Proposition 4.2.3 of [10])

$$
\begin{gathered}
u \varepsilon L^{2}\left(0, T ; H^{(2 r+1) \ell}(\Omega)\right), \\
\frac{\partial u}{\partial t} \varepsilon H^{2 r \ell, 2 r}(Q) \subset L^{2}\left(0, T ; H^{2 r \ell}(\Omega)\right), \\
\frac{\partial^{2} u}{\partial t^{2}} \varepsilon H^{(2 r-1) \ell, 2 r-1}(Q) \subset L^{2}\left(0, T ; H^{(2 r-1) \ell}(\Omega)\right) .
\end{gathered}
$$


Therefore

$$
u \varepsilon H^{2}\left(0, T ; H^{(2 r-1) \ell}(\Omega)\right),
$$

Theorem 4.1. Assume (4.4)-(4.8) hold and that

$$
0<1-\theta<\frac{1}{2(m-\ell)}
$$

Then

$$
\begin{aligned}
\left|\frac{\partial u_{\epsilon}}{\partial t}-\frac{\partial u}{\partial t}\right|_{L^{\infty}\left(0, T^{\prime} L^{2}(\Omega)\right)} & +\left|u_{\epsilon}-u\right|_{L^{\infty}\left(0, T^{\prime} ; H^{\ell(\Omega))}\right)} \\
& \leqq C\left\{\left[\max \left(\alpha_{\epsilon}{ }^{1 / 2}, \beta_{\epsilon}{ }^{1 / 2}\right)\right]^{1-\theta} \cdot|u|_{H^{2}\left(0, T ; H^{(1-\theta) m+\theta} \ell(\Omega)\right)}+\left|f_{\epsilon}-f\right|_{L^{2}(\theta)}\right\}
\end{aligned}
$$

Proof. Since

$$
H_{0}{ }^{s}(\Omega)=\left\{u: u \varepsilon H^{s}(\Omega),\left(\frac{\partial}{\partial n}\right)^{i} u=0 \quad \text { on } \quad \partial \Omega, \quad 0 \leqq j<s-\frac{1}{2}\right\}
$$

([10, Theorem 1.11.5]) we have

$$
H_{0}{ }^{\ell}(\Omega) \cap H^{(2 r-1) \ell}(\Omega) \subset H_{0}{ }^{(1-\theta) m+\theta \ell}(\Omega)
$$

provided

$$
(1-\theta) m+\theta \ell-\frac{1}{2}<\ell \text { and }(1-\theta) m+\theta \ell \leqq(2 r-1) \ell .
$$

Both of these conditions on $\theta$ are satisfied because of (4.10) and our choice of $r$ in (4.8). In addition, we have

$$
\left[H_{0}{ }^{m}(\Omega), H_{0}^{\ell}(\Omega)\right]_{\theta}=H_{0}^{(1-\theta) m+\theta \ell}(\Omega)
$$

with equivalent norms ([10, Theorem 1.11.6]; c.f. [5]. $(1-\theta) m+\theta \ell \neq$ integer $+1 / 2$ because of $(4.10))$. Since $u(t) \varepsilon H_{0}{ }^{\ell}(\Omega)$ for $0 \leqq t<T$ and $(\partial / \partial n)^{k} \varepsilon \&\left(H^{s}(\Omega)\right.$, $\left.H^{s-k-1 / 2}(\Gamma)\right)$ for $s>k+1 / 2$, we conclude from (4.9) that

$$
u \varepsilon H^{2}\left(0, T ; H_{0}^{\ell}(\Omega)\right) \text {. }
$$

Finally, since the injection of $H^{(2 r-1) \ell}(\Omega)$ into $H^{(1-\theta) m+\theta \ell}(\Omega)$ is continuous, we may conclude from (4.9) and (4.11)-(4.13) that

$$
u \varepsilon H^{2}\left(0, T ;\left[H_{0}{ }^{m}(\Omega), H_{0}{ }^{\ell}(\Omega)\right]_{\theta}\right) .
$$

The theorem now follows from (4.14) and Corollary 3.1.

5. Appendix. The purpose of this section is to prove an existence-uniqueness theorem for a class of problems which contains (3.1).

Let $V$ be a reflexive and separable Banach space and $1<p<\infty$. Let

$$
H^{n, p}(0, T ; V)=\left\{u: u, u^{\prime}, \cdots, u^{(n)} \varepsilon L^{p}(0, T ; V)\right\} .
$$


It is a Banach space under the norm

$$
|u|_{n, p}=\left[\int_{0}^{T} \sum_{i=0}^{n}\left|u^{(i)}(t)\right|_{V}^{p} d t\right]^{1 / p}
$$

Let $\left\{Q_{i}(t): 0<t<T, j=0,1, \cdots, n\right\}$ be a family of bounded linear operators from $V$ into $V^{\prime}$. We consider the problem

$$
\begin{gathered}
\sum_{i=0}^{n} a_{i}(t) u^{(j)}=f \varepsilon L^{p}\left(0, T ; V^{\prime}\right), \\
u_{j}(0)=x_{i} \varepsilon V, \quad j=0,1, \cdots, n-1, \\
u \varepsilon H^{n, p}(0, T ; V) .
\end{gathered}
$$

We assume

(5.4) For each $v \varepsilon V$ the map $t \rightarrow Q_{i}(t) v:(0, T) \rightarrow V^{\prime}$ is measurable, $j=0$, $1, \cdots, n$ (under our hypotheses on $V$, the concepts of weak and strong measurability coincide).

(5.5) For almost all $t, a_{n}(t)$ is a bijection of $V$ onto $V^{\prime}$ and

$$
\underset{(0, T)}{\operatorname{ess} \sup }\left|Q_{n}^{-1}(t)\right|_{\mathscr{L}\left(V^{\prime} ; V\right)}<\infty .
$$

It follows from (5.4) and (5.5) that the maps $t \rightarrow\left(Q_{n}\right)^{-1}(t) v^{\prime}\left(v^{\prime} \varepsilon V^{\prime}\right)$ and $t \rightarrow\left(Q_{n}\right)^{-1}(t) Q_{j}(t) v(v \varepsilon V, j=0,1, \cdots, n-\cdot 1)$ of $(0, T)$ into $V$ are measurable (c.f. [11, Lemmas 3.1 and 3.2]). We assume

$$
\underset{(0, T)}{\operatorname{ess} \sup _{(1)}}\left|{Q_{n}}^{-1}(t) Q_{i}(t)\right|_{\mathcal{L}(V, V)}<\infty, \quad j=0,1, \cdots, n-1 .
$$

Theorem 5.1. Assume (5.4), (5.5) and (5.6). Then for each $f \varepsilon L^{p}\left(0, T ; V^{\prime}\right)$ and $\left\{x_{i}\right\} \& V^{n}$ the problem (5.1)-(5.3) has a unique solution. Furthermore the map $\left[f,\left\{x_{j}\right\}\right] \rightarrow u$ of $L^{p}\left(0, T ; V^{\prime}\right) \times V^{n}$ into $H^{n-1, p}(0, T ; V)$ is continuous.

Proof. (5.1)-(5.3) is equivalent to

$$
\begin{gathered}
u^{(n)}+\sum_{j=0}^{n-1} B_{j}(t) u^{(j)}=g \varepsilon L^{p}(0, T ; V), \\
u^{(j)}(0)=x_{i} \varepsilon V, \quad j=0,1, \cdots, n-1, \\
u \varepsilon H^{n, p}(0, T ; V)
\end{gathered}
$$

where

$$
\Theta_{i}(t)=Q_{n}^{-1}(t) Q_{i}(t)
$$

and

$$
g(t)=a_{n}^{-1}(t) f(t) .
$$

Introducing $u_{i}=u^{(i)}(0 \leqq j \leqq n-1),(5.7)-(5.9)$ is equivalent to 


$$
\begin{gathered}
u_{i}^{\prime}-u_{i+1}=0, \quad j=0,1, \cdots, n-2, \\
u_{n-1}{ }^{\prime}+\sum_{i=0}^{n-1} \AA_{i}(t) u_{i}=g, \\
u_{i}(0)=x_{i}, \quad j=0,1, \cdots, n-1, \\
u_{i} \varepsilon H^{n-i, p}(0, T ; V), \quad j=0,1, \cdots, n-1 .
\end{gathered}
$$

Remark. The map $u \rightarrow \wp_{i}(\cdot) u(\cdot)(j=0,1, \cdots, n-1)$ is a bounded linear map of $L^{p}(0, T ; V)$ into itself. Thus, in view of the nature of the system (5.10), (5.11), it suffices to require, in place of (5.13), that

$$
u_{i} \varepsilon L^{p}(0, T ; V), \quad j=0,1, \cdots, n-1 .
$$

Now setting

$$
U=\left(\begin{array}{c}
u_{0} \\
u_{1} \\
\vdots \\
u_{n-1}
\end{array}\right), \quad X=\left(\begin{array}{c}
x_{0} \\
x_{1} \\
\vdots \\
x_{n-1}
\end{array}\right), \quad G=\left(\begin{array}{c}
0 \\
\vdots \\
0 \\
g
\end{array}\right)
$$

and

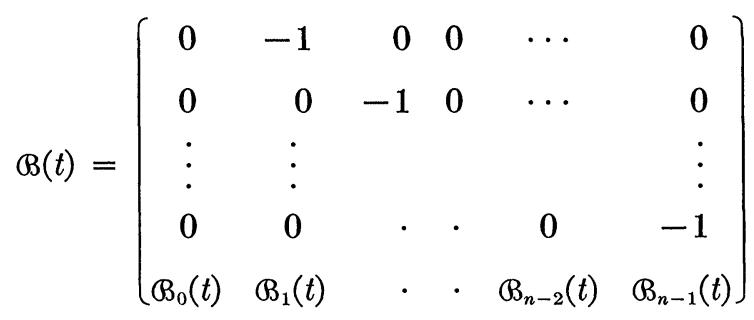

we see that $(5.10)-(5.12),(5.14)$ is equivalent to

$$
\begin{gathered}
U^{\prime}+ß(t) U=G, \\
U(0)=X, \quad U \varepsilon L^{p}\left(0, T ; V^{n}\right) .
\end{gathered}
$$

Theorem 5.1 is therefore a consequence of

Theorem 5.2. Assume (5.4), (5.5) and (5.6). Then for each $G \varepsilon L^{p}\left(0, T ; V^{n}\right)$ and $X \boldsymbol{\varepsilon} V^{n}$ the problem (5.15), (5.16) has a unique solution and the map $[G, X] \rightarrow U$ of $L^{p}\left(0, T ; V^{n}\right) \times V^{n}$ into $L^{p}\left(0, T ; V^{n}\right)$ is continuous.

Proof. Let $\mho=L^{p}\left(0, T ; V^{n}\right)$ and, for $U \varepsilon v$ define $(B U)(t)=-\beta(t) U(t)$. Then $B \varepsilon \&(\mathcal{V}, \mathcal{V})$. Let $\Lambda=-d / d t$ with $D(\Lambda)=\left\{U: U, U^{\prime} \varepsilon \mathcal{V}, U(0)=0\right\}$. Then $\Lambda$ is the generator of a continuous semigroup of contractions on $\mathcal{V}$. By a well known result on perturbation of semigroups, $\Lambda+B$ on $D(\Lambda)$ is the generator of a continuous semigroup of bounded linear operators on $\vartheta$. Thus 
$(\Lambda+B-k I)^{-1}$ exists for all sufficiently large positive values of $k$ as an everywhere defined, bounded linear operator on $\mathcal{V}$. Thus the problem

$$
\begin{gathered}
V^{\prime}+(B(t)+k) V=H \varepsilon L^{p}\left(0, T ; V^{n}\right), \\
V(0)=0, \quad V \varepsilon L^{p}\left(0, T ; V^{n}\right)
\end{gathered}
$$

has a unique solution and the map $H \rightarrow V$ of $L^{p}\left(0, T ; V^{n}\right)$ into itself is continuous. Theorem 5.2 now follows upon setting $U=e^{k t} V+X$.

\section{REFERENCES}

1. P. L. Butzer \& H. Berens, Semi-groups of operators and approximation, Springer-Verlag, New York, 1967.

2. A. Friedman, Singular perturbations for partial differential equations, Arch. Rational Mech. Anal. 29 (1968), 289-303.

3. W. M. Greenlee, Rate of convergence in singular perturbations, Ann. Inst. Fourier (Grenoble) 18 (1968), 135-191.

4. - Singular perturbation theorems for semibounded operators, J. Functional Anal. 8 (1971), 469-491.

5. P. Grisvard, Caractérisation de quelques espaces d'interpolation, Arch. Rational Anal. 25 (1967), 40-63.

6. D. Huet, Perturbations singulières et régularité, C. R. Acad, Sci. Paris Ser. A-B 266 (1968), A 924-A 926, A $1237-$ A 1239.

7. J. Lagnese, Approximation of solutions of differential equations in Hilbert space, J. Math. Soc. Japan 25 (1973), 132-143.

8. - Rate of convergence in a class of singular perturbations, J. Functional Anal. 13 (1973), 302-316.

9. J. L. Lions, Espaces intermédiaires entre espaces hilbertiens et applications, Bull. Math. Soc. Sci. Math. Phys. R. P. Roumaine (N.S.) 2 (1958), 419-432.

10. J. L. Lions \& E. MAgenes, Non-homogeneous boundary value problems and applications, Vols. I \& II, Springer-Verlag, New York, 1972.

11. R. E. Showalter, Existence and representation theorems for a semilinear Sobolev equation in Banach space, SIAM J. Math. Anal. 3 (1972), 527-543.

12. S. L. Soвolev, Some new problems in mathematical physics, Izv. Akad. Nauk SSSR Ser. Mat., 18 (1954), 3-50. 